\title{
Niederländisch toch und Deutsch doch: Gleich oder doch nicht ganz? Ad Foolen* (Nijmegen)
}

\begin{abstract}
In this paper, a semantic analysis is presented of the Dutch particle toch and its German cognate doch. On the formal level they may appear as either adverb, conjunction or interjection, and they also occur in different sentence types. It is argued that the core meaning of both particles is the same. The range of uses differs, however, between the two languages. For example, German doch can be used as an 'answering particle' in reaction to a negative statement or question. This is not possible in Dutch. The core meaning of the particles can be explicated in terms of three steps: the utterance with toch/doch represents a positive step. This step is preceded (implicitly or explicitly) by a negative step, which is in turn preceded by a positive step wich is identical to the actual positive step. For certain uses of toch and doch, however, we have to assume a modification of this general schema. A Dutch novel (by Maarten 't Hart, 1983) and its German translation was used as a corpus. The original text contained 270 occurrences of toch, and the translation 230 doch's.
\end{abstract}

\section{$1 \quad$ Partikeln im Niederländischen und Deutschen}

Sowohl Niederländisch als auch Deutsch sind partikelreiche Sprachen. Es gibt verschiedene Partikeln, die sich, auf den ersten Blick, direkt entsprechen: dan und denn, ja und ja, toch und doch, wel und wohl. Das Niederländische hat sogar ein paar Partikeln aus dem Deutschen übernommen: sowieso und überhaupt sind regelmäßig in niederländischen Äußerungen zu finden.

Diese scheinbare Ähnlichkeit hat wahrscheinlich dazu beigetragen, dass sich keine systematische längerfristige Forschungstradition entwickelt hat, in der die Partikeln dieser beiden Sprachen kontrastiv erforscht werden. Es gibt keine zweisprachigen Partikelwörterbücher, wie sie z. B. für Deutsch-Englisch (König et al.1990) und Deutsch-Französisch (Métrich et al.1996-2002) geschrieben wurden. In den 80er Jahren gab es ein paar vereinzelte Ansätze zur kontrastiven Partikelanalyse Niederländisch-Deutsch, u. a. Abraham (1981) und Westheide (1989), aber diese Ansätze fanden bis jetzt keine Fortführung. Und doch liegt

* Ich danke Susanne Requardt für Diskussion und Unterstützung. 
hier eine interessante Herausforderung, gerade wegen der scheinbaren Ähnlichkeit. Westheide (1989: 295) betonte zu Recht, dass ".. die Verwandschaft der Formen und die räumliche Nähe über die grundsätzlichen Unterschiede in den Sprechökonomien hinwegtäuschen, was nicht selten im Sprachkontakt zu erheblichen Mißverständnissen führt". Beim Lernen der anderen Sprache ist es nur zu verführerisch, sich von dem Transparenzprinzip, 'eine Form eine Bedeutung', leiten zu lassen, wie Abraham (1981: 186) es formuliert: "[S]o lange es geht, hält der Lerner an der vereinfachenden Hypothese fest, daß eine Lautform für bedeutungsverwandte oder bedeutungsgleiche Lexeme steht".

Angesichts dieser Forschungslage ist es umso erfreulicher, dass Wenzel (2002) in ihrer detaillierten Studie zur niederländischen Lernersprache von Deutschen den Partikeln die gebührende Aufmerksamkeit geschenkt hat. Um nur ein Beispiel zu nennen: Sie stellte fest, dass die Lerner relativ häufig das Partikelcluster toch wel benutzen (Wenzel 2002: 187). Diese 'Strategie' wird verständlich, wenn man weiß, dass D. doch in bestimmten Kontexten mit N. wel übersetzt werden soll. Die Lerner benutzen mit toch wel eine Art Zwischenform, ein Partikelcluster, das an sich auch im Niederländischen benutzt wird, aber bei den Lernern auffällig häufig vorkommt.

Es ist zu hoffen, dass Wenzels Studie der Auftakt zur erneuten Partikelforschung Deutsch-Niederländisch bedeutet, und zwar in beiden Richtungen. Dabei sollten verschiedene Forschungsmethoden einander ergänzen. Neben der von Wenzel angewandten Methode der Analyse von Lernerkorpora bleibt die traditionellere grammatisch-kontrastive Analyse auf der Ebene des Sprachsystems wertvoll (wie schon Weydt 1969 mit seinem Vergleich des Deutschen und Französischen gezeigt hat, cf. auch Fischer und Drescher 1996). In den jetzigen Beitrag möchte ich einen Übersetzungskorpus einbeziehen, um zu sehen, inwieweit daraus kontrastive und einzelsprachige Einsichten gewonnen werden können.

\section{2 Übersetzungskorpora in der Partikelforschung}

Nach Aijmer und Simon-Vandenbergen (2002, Par. 4) sind gerade fiktionale Texte besonders dafür geeignet, in kontrastive Partikelforschung einbezogen zu werden. Autoren von fiktionalen Texten benutzen relativ häufig Partikeln zur Charakterisierung von Personen und Situationen. Nicht nur kontrastive Einsichten können auf diese Weise gewonnen werden, auch für die einzelsprachliche Analyse kann der Vergleich wertvoll sein: "If a discourse particle in the source language is shown to have been translated by a range of items in the target language, this finding forces one to consider the contextual meanings which the source language item adopts". 
Weydt (2001: 796-98) warnt allerdings vor den Gefahren dieser Methode: "In Wirklichkeit stellt sich das Äquivalenzproblem nicht auf der Ebene des Wortes, nicht einmal auf der des Satzes, sondern auf der des Textes. Was zu übersetzen ist, sind nicht Partikeln, sondern es sind Texte (die Partikeln enthalten)" (S. 797). Dem ist zwar im Allgemeinen zuzustimmen, aber gerade bei eng verwandten Sprachen findet sich die Äquivalenz oft doch auf der Ebene des Wortes, sodass ein Vergleich auf der Ebene möglich und vielleicht auch ergiebig ist. MitchellSchuitevoerder (2001: 22) stellt fest, dass in der Übersetzungstheorie das Übersetzen auf Wortniveau nicht allgemein verpönt ist: "Newmark (1981) would not consider a pragmatic approach in conflict with a literal word-for-word translation: 'Literal translation is correct and must not be avoided, if it secures referential and pragmatic equivalence to the original"'.

In den letzten zehn Jahren wurden viele niederländische Autoren ins Deutsche übersetzt: Cees Noteboom, Harry Mulisch, Maarten 't Hart, Tessa de Loo und Margriet de Moor sind nur einige Namen, die inzwischen beim lesenden Publikum in Deutschland bekannt sind. Für den jetzigen Beitrag habe ich einen Roman von Maarten 't Hart aus dem Jahr 1983 ausgewählt (De kroongetuige), der 2001 ins Deutsche übersetzt wurde (Die schwarzen Vögel). Maarten 't Hart ist dafür bekannt, dass er natürliche Dialoge schreibt, was frequenten Gebrauch von Partikeln erwarten lässt. In dem ausgewählten Text waren in der Tat genügend Partikeln $\mathrm{zu}$ finden. Ich habe mich für diese Studie auf toch konzentriert. Der niederländische Text zählt 212 Seiten, darin kommt diese Partikel 270-mal vor, also mehr als einmal pro Seite. 179 davon, also ungefähr zwei Drittel, wurden mit doch übersetzt, was darauf hinweist, dass die Übersetzerin womöglich 'direkt' übersetzt hat. In 91 Fällen hat sie allerdings eine andere Übersetzung vorgezogen. Umgekehrt kommt im deutschen Text 52-mal doch vor, ohne dass dem ein toch zu Grunde liegt. Die deutsche Version enthält insgesamt 231-mal doch, 39 weniger als toch in der niederländischen Version. Zum Teil ist das die Folge davon, dass bei der Übersetzung die Partikel in 32 Fällen 'negiert' wurde, das heisst, dass sie weder durch doch noch auf eine andere Weise im deutschen Text eine Entsprechung fand.

Bevor ich zu Einzelheiten des Vergleichs komme, gehe ich kurz auf die Partikelbeschreibung im Allgemeinen und dann auf die Analyse von toch und doch im Besonderen ein.

\section{Beschreibungsebenen für Partikelbedeutungen}

Die Bedeutungsanalyse für Partikeln soll auf mehreren Abstraktionsebenen stattfinden (cf. Foolen 1989). Weydt (2001: 794-95) unterscheidet die pragmatische Bedeutung von der linguistisch konventionalisierten Bedeutung. Die pragmatische Bedeutung betrifft Effekte, die auftreten, wenn eine Partikel in 
einem bestimmten Kontext verwendet wird. Nach Weydt handelt es sich dabei typischerweise um expressive und interaktionelle Effekte, wie Freundlichkeit, Ungeduld, Staunen. Was die konventionalisierte Bedeutung angeht, unterscheidet Weydt zwischen Grundbedeutung und 'primärer' Bedeutung.

Weydt hat bei seiner Partikelforschung über die Jahre hinweg an der Idee einer Grundbedeutung festgehalten: "Jeder Partikel lässt sich eine konstante Bedeutung zuordnen. Diese ist ein Ensemble von semantischen Merkmalen, das bei jedem Vorkommen der betreffenden Partikel auftritt" (2001: 794). Dieses Konzept, das auch als 'übergreifende Bedeutung' oder 'core meaning' (Aijmer 2002: 23) bezeichnet wird, ist nicht unumstritten, aber ich werde es hier, in eine bestimmte theoretische Perspektive eingebettet, beibehalten.

Von der Grundbedeutung sind die konventionalisierten Gebrauchsweisen zu unterscheiden, die in Weydt (2001) als 'primäre' Bedeutungen bezeichnet werden (S. 794): "Die Grundbedeutung wird in verschiedenen Kontexten differenziert. Diese spezifischeren Bedeutungen bleiben damit immer noch primär. (...) Man findet solche Varianten oft in den ein- oder zweisprachigen Lexika, differenziert, und numeriert".

Meiner Auffassung nach soll die Annahme solcher Beschreibungsebenen nicht heißen, dass sie für jede Partikel gleich relevant sind. Bei bestimmten Partikeln können die Gebrauchsweisen so ausgeprägt sein, dass eine gemeinsame Grundbedeutung wenig prägnant ist. Die allgemeinen Überlegungen, die Langacker im Rahmen der kognitiven Grammatik zu Abstraktionsebenen in der Bedeutungsbeschreibung angestellt hat, treffen m. E. auch auf Partikeln zu. Langacker geht in seiner Analyse 'usage based', 'bottom up' vor. Die Gebrauchsweisen, die er für ein Wort oder eine Konstruktion unterscheidet, werden in einem Netzwerk zu einander in Verbindung gebracht. "Often (but not necessarily always), a highly abstract schema emerges which all other senses instantiate as special cases" (Langacker, to appear). Langacker betrachtet also die Grundbedeutung als eine Abstraktion der Gebrauchsweisen, eine Abstraktion, die nicht notwendigerweise immer deskriptiv angebracht sein muss, und auch nicht unbedingt von allen Sprechern einer Sprache vollzogen wird.

\section{Die Grundbedeutung von toch und doch}

Nicht alle Autoren, die sich mit der Analyse von doch beschäftigt haben, gehen von einer Grundbedeutung aus. Franck (1980: 172) betrachtet doch als "mehrfach homonym". Bei den Autoren, die eine Grundbedeutung annehmen, gibt es zwei Auffassungen, die ich als 'Zweischrittanalyse' und 'Dreischrittanalyse' bezeichnen werde.

Die Zweischrittanalysen finden sich eher bei empirisch orientierten Studien. Weydt (1969: 39) formuliert diese Sichtweise folgendermaßen: "Als allen Be- 
deutungen gemeinsames Element ist eine leicht adversative Bedeutung auszumachen. Der Satz mit doch enthält einen Gegensatz zu der bis dahin angenommenen Vorstellung. Die neue Vorstellung soll vom Hörer übernommen werden. 'Das mußt Du doch zugeben' (also ist das vorher Gesagte falsch)". Auch in Kleine deutsche Partikellehre wird 'leichte Adversativität' als zentrale Komponente der übergreifenden Bedeutung hervorgehoben (Weydt et al.: 162). Die übergreifende Bedeutung wird zwar zunächst nur für die 'Abtönungsvarianten' formuliert, soll aber auch den semantischen Zusammenhang mit 'Homonymen' (Antwortpartikel, Konjunktion) erfassen (Weydt et al. 1983: 159).

Hentschel (1986: 148) spricht statt von Adversativität von Widerspruch: "Doch drückt einen Widerspruch zwischen zwei Bezugspunkten aus. Zumindest einer von beiden wird dabei als dem Hörer bekannt vorausgesetzt". Bastert (1985: 118) schlägt auf Grund ihrer detaillierten lexikographischen Studie den folgenden Lexikoneintrag vor: "Durch die Äußerung mit doch hebt der Sprecher einen tatsächlichen oder vermuteten Gegensatz zu einer vorangegangenen Äußerung, dem Vortext o.ä. hervor. Die Äußerung erscheint ihm als berechtigt". Auch die zweisprachigen Partikelwörterbücher übernehmen diese Sichtweise; nach König et al. (1990: 58) weist doch "auf einen Gegensatz zwischen zwei Sachverhalten hin". Und Métrich et al. (1996: 148) ziehen nach der Analyse der verschiedenen Gebrauchsweisen von doch die Bilanz, dass die 'valeur générale' von diesem Wort folgendermaßen formuliert werden kann: "On peut dire que doch marque d'une façon générale une relation adversative en ce sens qu'il exprime ou suggère une contradiction entre l'énoncé où il figure (éventuellement réduit à doch lui-même) et l'énoncé précédent, un contenu de pensée implicite ou un comportement".

Der Zweischrittanalyse möchte ich die Dreischrittanalyse gegenüberstellen, die schon sehr früh (1939) vom japanischen Germanisten Sekiguchi ${ }^{1}$ formuliert wurde, und die ich daher auch ab und zu als die Sekiguchi-Analyse bezeichnen werde (hier zitiert nach der Übersetzung aus 1977: 6):

Doch heißt, eine verneinende Aussage über denselben Sachverhalt als stillschweigend anerkannt voraussetzen und über deren Verneinung zur ursprünglichen Bejahung zurückkehren. (...) Es handelt sich kurz um zwei Bewegungen, die drei Stationen durchlaufen. Bei Es hat doch geschneit verhält es sich genauso. (...):

(1. Stufe = Bejahung): Man kann eigentlich davon ausgehen, daß es jedes Jahr schneit. (Selbstverständlichkeit).

(2. Stufe = Bewegung zur Verneinung): Könnte es vielleicht trotzdem sein, daß es in diesem Jahr nicht zum Schneien kommt?

${ }^{1} \mathrm{Zu}$ der Germanistik in Japan, cf. Eijirô (2002), im Besonderen die Seiten 10-12 zu Sekiguchi. 
(3. Stufe $=$ Rückkehr zur Bejahung): Nein, es hat doch geschneit. (...)

Es darf keines der drei Momente (...) fehlen. Und je nachdem, ob sie jeweils auf einen bestimmten Typ beschränkt sind oder in den Vordergrund bzw. in den

Hintergrund treten, ergeben sich verschiedene Verwendungsweisen von doch.

In der niederländischen Partikelforschung ist toch von verschiedenen Autoren analysiert worden. Es ist hier nicht möglich, allen Standpunkten gerecht zu werden. Ich möchte nur hervorheben, dass die von Sekiguchi formulierte Sichtweise auch hier vertreten wurde. Elffers (1992) nimmt 'Persistenz' als Grundbedeutung für toch an, und in Foolen (1994) wurde der Vorschlag von Sekiguchi in Bezug auf die niederländischen Daten besprochen.

Zur deutschsprachigen Literatur zurückkehrend, finden wir diese Ansicht explizit vertreten in Graefen (2000: 3): "Zusammenfassend gesagt, tritt doch an charakteristischen Stellen auf, nämlich dort, wo ein Sprecher eine im Diskurs zunächst verbalisierte oder präsupponierte, aber dann in Frage gestellte Auffassung aufrechterhalten will. Mit dem Element doch lenkt der Sprecher den Verstehensprozess so, dass dieser den propositionalen Gehalt als eine Rekurrenz des Sprechers auf Vorhergehendes oder Vorausgesetztes deutet".

Wenn Sekiguchi sagt, dass keines der drei Momente fehlen darf, dann verstehe ich das so, dass sie 'irgendwie' anwesend sein müssen, explizit oder implizit. Der zweite Schritt, sei er explizit oder implizit, ist immer der negative Schritt, und ich gehe davon aus, dass eine Negation immer einen positiven Vorgängerschritt voraussetzt, der auch wiederum explizit oder implizit sein kann.

Bis jetzt hat die Partikelforschung es versäumt, den beiden Vorschlägen auf den Grund zu gehen. Ich werde das hier auch nur beschränkt tun können, werde aber bei der folgenden Besprechung der verschiedenen Gebrauchsweisen prüfen, welche Grundbedeutung jeweils am besten passt.

\section{Gebrauchsweisen}

So sehr ich auch mit Graefen einer Meinung bin, dass es wichtig ist, die Grundbedeutung einer Partikel präzise zu erfassen, so wenig konstruktiv scheint mir die Art und Weise, wie sie den Stellenwert der Beschreibung der Gebrauchsweisen darstellt. In der 'empirischen Konkretion' der Partikelforschung, wie sie es nennt (S. 1), sieht sie sogar einen "Grund für ihre derzeitige Stagnation" (S. 2). Und auch für den Fremdsprachenunterricht rät sie, der Grundbedeutung eine zentrale Rolle beizumessen (S. 9): "Mir scheint, dass gerade für den Fremdsprachenerwerb das didaktische Ziel darin bestehen sollte, statt einer langen Liste von grammatisch modifizierten Teilbedeutungen einen flexiblen, situationsadäquaten Umgang mit - relativ abstrakt gefaßten - Grundbedeutungen zu erlernen". 
Dem ist entgegenzuhalten, dass die Gebrauchsweisen auf jeden Fall zum Sprachwissen des 'native speakers' gehören, cf. Janssen (1995: 266): "... the language user has a highly detailed knowledge of when linguistic elements can be employed and when they cannot. And it is this very kind of knowledge that any language learner needs to have. The abstract description is obviously not enough to prevent him from making mistakes". Eine Beschränkung des Fremdsprachenunterrichts oder der Bedeutungsbeschreibung auf die Grundbedeutung, wie sie von strengen 'Monosemisten' vertreten wird, wäre darum verfehlt, genauso wie es unbefriedigend ist, die Beschreibung nur aus einer Liste von Gebrauchsweisen bestehen zu lassen. Eine integrierte Sprachbeschreibung erfordert eine ausgewogene Analyse auf beiden Ebenen.

Die schwierige Frage ist allerdings, nach welchen Kriterien die Gebrauchsweisen unterschieden werden müssen. Bei doch können wir unterscheiden nach Wortart oder Funktionsklasse (Antwortpartikel, Konjunktion, Adverb, Modalpartikel), nach Betonung (betontes und unbetontes doch), nach Satztyp (affirmativ, direktiv, fragend, exklamativ), Sprechhandlung oder nach Position (im affirmativen Satz kann das Adverb doch im Mittelfeld, aber auch an der ersten Satzstelle stehen). Das gewählte Kriterium muss auch nicht unbedingt für alle Partikeln dasselbe sein, oder vielleicht nicht einmal für alle Gebrauchsweisen einer bestimmten Partikel. Die empirischen Gegebenheiten spielen hier eine entscheidende Rolle.

Wie wir vorher gesehen haben, schlägt Sekiguchi vor, die Art der Gegebenheit der drei 'Momente' als Grundlage für die Unterscheidung der Gebrauchsweisen zu benutzen. Ich zitiere noch einmal das Ende des oben stehenden längeren $\mathrm{Zi}$ tats: "Es darf keines der drei Momente (...) fehlen. Und je nachdem, ob sie jeweils auf einen bestimmten Typ beschränkt sind oder in den Vordergrund bzw. in den Hintergrund treten, ergeben sich verschiedene Verwendungsweisen von doch." Was er genau mit 'auf einen bestimmten Typ beschränkt' meint, ist mir nicht ganz klar, aber Vordergrund bzw. Hintergrund hat wohl damit zu tun, ob die Momente explizit oder implizit im Diskurs gegeben sind. Hiermit haben wir noch ein zusätzliches Kriterium zur Unterscheidung von Gebrauchsweisen, diesmal eher konversationeller Art.

Genau zu diesem Punkt, bei der Frage, welche Gebrauchsweisen unterschieden werden sollen, kann eine kontrastive Analyse behilflich sein. Wenn doch in bestimmten Kontexten systematisch auf eine spezifische Weise übersetzt wird, ist das Anlass, ernsthaft zu überlegen, eine eigene Gebrauchsweise anzunehmen. Im Folgenden werde ich mit Hilfe des Übersetzungsmaterials ein paar Gebrauchsweisen besprechen. 


\section{Doch als Reaktionspartikel}

Graefen (2000: 4) sieht die "pragmatische Grundbedeutung [von doch] ... am klarsten im Falle der Antwortpartikel realisiert ... Von hier aus hat eine Ausweitung des Gebrauchs von doch auf andere Sprechhandlungstypen oder Handlungsmuster ... stattgefunden". Die letztere Behauptung kann nur durch eine diachrone Untersuchung geprüft werden, dem ersten Teil ist aber, wenn man der Sekiguchi-Analyse anhängt, zuzustimmen. Terminologisch würde ich statt 'Antwortpartikel' lieber 'Reaktionspartikel' benutzen, da dieses doch nicht nur nach Fragen vorkommt. Wenn A sagt, dass Harald 65 wird, kann B mit $j a$ zustimmen, oder mit nein verneinen. Wenn B mit nein reagiert, kann A im folgenden Dialogschritt mit doch auf seine Ausgangsbehauptung zurückgreifen. Ein ähnliches Reaktionssystem findet sich auch im Französischen (oui, non, si), cf. Kerbrat-Orecchioni (2001), die übrigens zeigt, dass die Anwendung des Systems im Sprachgebrauch nicht einfach und mechanisch stattfindet, etwa in der Weise, dass ein negativer Vorgängersatz automatisch si hervorruft. Van Valin (1975) hat auch für doch darauf hingewiesen. So kontrastiert er 'Hans behauptet, dass Jan nicht gekommen ist' mit 'Hans behauptet, dass Jan nicht gekommen sei'. Im zweiten Fall, mit Konjunktiv I im Nebensatz, wäre 'Doch, er ist gekommen' unangebracht (S. 20).

Im Niederländischen fehlt ein solches eindeutiges Reaktionssystem. Es ist nicht verwunderlich, dass einer der von Wenzel untersuchten Lerner auf die Bemerkung Die ken je ook niet ('Die kennst du auch nicht') mit Doch! reagiert (Wenzel 2002: 191), ein Transfer aus dem Deutschen. Wenn wir uns die Übersetzung von 't Harts Roman anschauen, dann sehen wir, dass die Reaktionspartikel doch zurückgeht auf ja, wel, oder jawel im Original. Wel ist zu analysieren als ein betontes $j a$, so dass die Dreischrittanalyse auch für wel angebracht scheint.

\section{$7 \quad$ Doch als Konjunktion}

Im Deutschen wird doch als nebenordnende Konjunktion benutzt. Alle Fälle der Konjunktion doch im untersuchten Text entsprechen im niederländischen Original der adversativen Konjunktion maar. In den meisten Fällen wird die Konjunktion maar mit aber übersetzt. Interessant ist die Frage, ob es hier ein Muster gibt: Wann wählt die Übersetzerin doch statt aber? Es fällt in diesem Korpus auf, dass auf die Konjunktion doch immer eine betonte, fokussierte Wortgruppe an der ersten Satzstelle folgt, cf. S. 97, wo über das Aussehen eines Mädchens gesprochen wird, in der Wirklichkeit und im Spiegelbild: "Das Mädchen sagte mir eigentlich nichts, ich sah zwar, dass sie ein wunderhübsches Gesicht hatte, aber ich fand es viel zu stark geschminkt. Doch im Spiegelbild wurde ihr Gesicht lieblich und fein ...". In diesem Beispiel kommt sowohl aber vor als auch doch, und nur nach doch folgt direkt eine kontrastierte Wortgruppe. Oder S. 27: 
"Sie sehnt sich leidenschaftlich nach Kindern, genau wie ich früher. Doch ich sehne mich nicht mehr ...". Meiner Interpretation nach wird hier ich zu sie in Kontrast gesetzt. Es fällt auf, dass im Original der Kontrast zwischen vroeger, 'früher' und $n u$, 'jetzt', konstruiert wird: "Ze verlangt hartstochtelijk naar kinderen, net als ik vroeger. Maar nu niet meer, ..." ('... genau wie ich früher. Doch jetzt nicht mehr').

Auf den ersten Blick passt die Gebrauchsweise als Konjunktion am besten zu einer Grundbedeutung, die von zwei Bezugsmomenten ausgeht. Es werden zwei Tatsachen kontrastiert, wobei die erste nicht negativ sein muss, und wobei die zweite nicht auf ein vorangehendes positives Moment zurückgreift. Betrachten wir ein weiteres Beispiel, S. 105: "Sogar Dein eigener Anwalt (...) scheint davon überzeugt zu sein, dass Du es getan hast. Doch einige finden es befremdlich, dass sie Dich festhalten ...". Im zweiten Konjunkt werden einige, die an die Unschuld glauben, kontrastiert zu den meisten, sogar zu dem Anwalt, die von der Schuld überzeugt sind. Es sieht so aus, dass doch in all diesen Fällen sich sehr stark der adversativen Konjunktion aber nähert. Dann stellt sich die Frage, ob für die adversative Konjunktion eine Zwei- oder Dreischrittanalyse angemessen wäre. Da bei den anderen Gebrauchsweisen von doch eine Dreischrittanalyse passend erscheint, möchte ich die hier auch für Adversativität vorschlagen: Konjunkt A ist der Fall (1. Schritt). Daraus könnte man folgern, dass Konjunkt B nicht der Fall ist (2. Schritt). Aber B ist der Fall (3. Schritt). Wenn sogar der Anwalt nicht mehr an der Unschuld glaubt, dann könnte man wohl denken, dass niemand mehr an die Unschuld glaubt, dass es also keine Personen geben wird, die daran glauben. Aber dennoch gibt es Personen, die daran glauben, wie im zweiten Konjunkt behauptet wird.

Obwohl auch dem konjunktionalen doch eine Dreischrittanalyse zu Grunde gelegt werden kann, ist es nicht so, dass im dritten Schritt auf Vorhergehendes zurückgegriffen wird. Aufgrund dieser Besonderheit kann für diese Gebrauchsweise eine Bedeutungsspezifizierung angenommen werden, die sich von der Grundbedeutung darin unterscheidet, dass der 3. Schritt nicht auf den 1. zurückgreift.

Abschließend möchte ich noch erwähnen, dass es im Originaltext eine Stelle gibt, an der toch als Konjunktion benutzt wird, S. 122: "onder die donkere, toch heldere hemel". Diese Gebrauchsweise von toch ist im modernen Niederländisch sehr selten, sie klingt altmodisch. In der Übersetzung wird aber benutzt (S. 177): "unter diesem dunklen, aber klaren Himmel". Die Observation, dass die Gebrauchskontexte einer Partikel sich diachron ändern, ist ein zusätzlicher Grund, in der Beschreibung verschiedene Gebrauchsweisen zu unterscheiden. 


\section{Doch als Konjunktionaladverb}

Sowohl toch als auch doch können als Konjunktionaladverb benutzt werden. Es wird durchgängig angenommen, dass das Konjunktionaladverb sowohl an der ersten Satzstelle als auch im Mittelfeld stehen kann. Bei der Auszählung habe ich diese Positionen getrennt gehalten. Toch besetzt 44-mal die erste Satzstelle. In der Übersetzung wird doch gewählt, aber noch häufiger dennoch (26-mal), was damit zu tun haben könnte, dass auf diese Weise Funktionsambiguität vermieden wird, bei doch am Satzanfang könnte es auch um die Konjunktion doch gehen.

Was die Relevanz für eine der beiden Gesamtbedeutungsanalysen betrifft, sieht es so aus, dass das Konjunktionaladverb immer drei Schritte impliziert. Die im Deutschen oft auftretende Übersetzung dennoch kann in diesem Zusammenhang als eine eher analytische Form der doppelten Bezugsnahme angesehen werden: den(n) bezieht sich auf die vorangehende Verneinung, noch auf die Ausgangssituation, die trotz des Zwischenfalles noch gilt. Einige Beispiele sollen die Dreischrittanalyse illustrieren. Beim ersten Beispiel geht es um ein doch an erster Satzstelle. Auf S. 16 im übersetzten Text wird darüber gestritten, ob die Versuchstiere sich nachts gegenseitig auffressen: "'Heute nacht sind sie untereinander wieder hübsch zugange gewesen', sagte er grantig. 'Das kann eigentlich nicht sein', sagte ich, 'sie hungern erst seit vier Tagen'. 'Nun, und doch sind sie wieder übereinander hergefallen, sieh dir das an ...". Diese Gebrauchsweise erinnert deutlich an die Reaktionspartikel doch.

Meibauer (1994: 104ff.) plädiert dafür, betontes doch im Mittelfeld nicht als Konjunktionaladverb, sondern als eine kontrastbetonte Modalpartikel zu analysieren. Aber bei den Beispielen im Korpus sehe ich keinen prinzipiellen Unterschied zwischen dem adverbialen doch an erster Stelle und im Mittelfeld, cf. folgendes Beispiel auf S. 61: "Er streckte mir seine Hand entgegen. Sollte ich sie schütteln? Ich hob meine Hand, hätte am liebsten an seinem Schlips gezogen, der jetzt unauffällig hin und her schwang, aber ich schüttelte ihm schließlich doch die Hand." Wiederum eine Dreischrittstruktur, wobei alle Momente explizit im Text anwesend sind. Ich gehe also vorläufig davon aus, dass im Deutschen alle Fälle von betontem doch, ob sie nun im Vorfeld oder Mittelfeld platziert sind, als dieselbe Gebrauchsweise betrachtet werden können.

Alle bis jetzt besprochenen Beispiele betreffen Aussagesätze. Nach Graefen (2000: 6) kann "doch nicht in der Sprechhandlung Frage auftreten", aber im untersuchten Text fand ich zwei Beispiele von toch/doch in Ja/Nein-Fragen (im ersten Beispiel geht es mir um das zweite toch/doch):

(S. 98) "Of toch wel, was ik toch geroerd omdat ze opeens van mij was gaan houden ..."

(S. 145) "Oder doch, war ich doch gerührt, weil sie mich auf einmal liebte" 
(S. 210) "Zou er dan toch iets zijn dat mij troosten kon voor de dood van die vrouw..."

(S. 303) "Sollte es doch etwas geben, was mich über den Tod dieser Frau hinwegtrösten könnte ..."

Diese Beispiele sind in der Tat vielleicht Fragen eines anderen Typs als Graefen im Sinn hat, es geht hier um reflexive Fragen, der Sprecher fragt sich, ob ein Gedanke, den er schon einmal hatte, möglicherweise richtig ist. Wie dem auch sei, wiederum erkennen wir hier leicht den Dreierschritt.

\section{Toch in der Bedeutung sowieso}

Im Niederländischen sieht es so aus, dass wir beim betonten toch differenzieren müssen. Es gibt Beispiele, bei denen betontes toch im Mittelfeld steht und nicht ohne Bedeutungsänderung an die erste Satzstelle gesetzt werden kann. In dem betreffenden Satz wird mit Hilfe von toch indiziert, dass ein Umstand 'ohnehin, sowieso' der Fall ist, was dann als Argument für eine vorgeschlagene Handlung gilt. Der Sprecher will sagen: Unter diesen Umständen ist die Handlung einfach nicht zurückzuweisen, Widerspruch ist zwecklos. Diese Gebrauchsweise ist nicht so frequent, aber im analysierten Text kommt sie 7-mal vor, und keine davon wird einfach mit doch übersetzt, was ein Indiz dafür ist, dass wir es hier in der Tat mit einer Gebrauchsweise zu tun haben, die eine eigene Bedeutung aufweist. Zwei Beispiele: "Nu ik hier toch ben zou ik graag eens wat rondkijken, kan dat?" (S. 54). "Nun, wo ich einmal hier bin, würde ich mich gern ein wenig umsehen. Geht das?" (S. 79). " 'Nee, nee,' zei hij, 'dat doe je niet, je blijft hier, ik moet toch zo weg'." (S. 76). " 'Nein, nein', sagte er, ,das tust du nicht, du bleibst hier, ich gehe sowieso gleich"' (S. 113). In einem Fall hat die Übersetzerin m. E. diese Gebrauchsweise nicht richtig erkannt (S. 95): "Trouwens, ik weet toch al wat de uitslag zal zijn", was übersetzt wurde mit "Obwohl ich doch schon weiß, wie das Ergebnis aussehen wird." Besser wäre: "Übrigens, ich weiß sowieso schon, wie das Ergebnis aussehen wird."

Obwohl wir es bei dieser Gebrauchsweise mit einer betonten Variante von toch zu tun haben, sind die Betonungsverhältnisse anders als beim Konjunktionaladverb. Bei der letztgenannten Gebrauchsweise verteilt sich die Betonung auf toch und eine weitere Konstituente im Satz, bei der begründenden Gebrauchsweise zieht toch die volle Betonung auf sich.

Auch wenn wir für die hier besprochene Gebrauchsweise von toch eine spezifizierte Bedeutung annehmen wollen, bleibt diese Spezifizierung im Rahmen der 'übergreifenden' Dreischrittanalyse. Die Übersetzung sowieso ist als analytische Wiedergabe der spezifischen Bedeutung zu analysieren: Es war so, und wie die Umstände auch sein mögen oder was auch immer passiert, es bleibt so, es ist 
nichts daran zu ändern. Der negative Schritt wird kategorisch verworfen, und das macht die besondere Bedeutung dieser Gebrauchsweise aus.

\section{Doch im begründenden Kontext}

Auch im Deutschen gibt es eine Gebrauchsweise von doch, die mit Begründung zu tun hat, die aber nicht mit der niederländischen Gebrauchsweise gleichgesetzt werden kann. Es handelt sich hier nicht um eine kategorische Aussage, es wird begründend auf ein Vorwissen zurückgegriffen.

Die hier zu unterscheidende Gebrauchsweise wurde u. a. in Borst (1985: 74-82) besprochen, der von 'doch im Kausalsatz' spricht. Im analysierten Text habe ich nur ein Beispiel gefunden (S. 46): "Fast ohne hinzusehen, konnte ich die Rille finden, bei der Cassio mit seiner Geschichte beginnt; hatte ich doch schon so oft diese Rille gesucht". Im niederländischen Text werden die beiden Sätze asyndetisch und ohne toch verbunden (S. 32): "Bijna blindelings kon ik de groef vinden waar Cassio zijn verhaal begint, had die groef al zo vaak gezocht". Doch indiziert hier, dass auf Grund des begründenden Satzes ('schon oft die Rille gesucht haben'), dasjenige, was begründet wird ('schnell die Rille finden können'), selbstverständlich ist. Angewandt auf das Beispiel: Wie vorher anzunehmen war (Schritt 1), konnte ich die Rille schnell finden (Schritt 3). Der Gedanke, dass ich die Rille nicht finden würde, sodass ich hinsehen müsste, (Schritt 2), wäre unangebracht.

Obwohl der Satztyp und auch der Textzusammenhang bei dieser Gebrauchsweise spezifisch sind, ist die eigene Bedeutung von doch hier nicht wesentlich anders als in der Dreischrittanalyse angenommen. Wenn die 'begründende' Bedeutung der Konstruktion als ganzer zugeschrieben werden kann, gibt es keinen Anlass hier eine spezifische Bedeutung für diese Gebrauchsweise anzunehmen. ${ }^{2}$

\section{Toch/doch als Modalpartikel}

Wenn toch/doch unbetont im Mittelfeld steht, wird generell von Modalpartikel (oder Abtönungspartikel) gesprochen. Der Satztyp kann variieren: assertiv, imperativ oder W-Frage. Zusammen geht es um 178 Vorkommen, womit die Gebrauchsweise als Modalpartikel weitaus die häufigste ist. Bei den 178 Fällen handelt es sich 140-mal um einen Assertivsatz, 18-mal um einen Imperativsatz, 11-mal um eine W-Frage, und 9 Beispiele habe ich als Exklamativsatz kategorisiert. Ich bespreche die verschiedenen Satztypen nacheinander.

Beim assertiven Satztyp verschiebt sich die Funktion der Äußerung oft in Richtung Frage. Die fragende Behauptung oder behauptende Frage wird dem Hörer

\footnotetext{
${ }^{2}$ Für weitere Diskussion dieser Gebrauchsweise verweise ich auf Önnerfors (1997) und Ormelius-Sandblom (1997).
} 
zur Beurteilung oder Beantwortung vorgelegt. Der Sprecher indiziert, dass er selber, oder, nach seiner Wahrnehmung der Gesprächspartner, von einem ersten sicheren Schritt aus in eine unsichere Phase geraten ist. Jetzt will er im dritten Schritt zur Sicherheit zurückkehren. In den meisten dieser Fälle wird toch mit doch übersetzt. Z. B. S. 120: "...ik geloof niet dat ik er ooit achter kom ...". "Je bent toch ook geen rechercheur. Dit is helemaal jouw werk niet". S. 174: "...ich glaube nicht, dass ich jemals dahinterkomme ...". "Du bist doch auch keine Kriminalbeamtin. Das ist überhaupt nicht deine Aufgabe".

Eine andere Übersetzung findet sich im folgenden Beispiel, S. 105: "Zal ik meegaan naar het vrouwenhuis?" Ik zweeg en ze begreep daaruit dat ik liever alleen ging. "Je kent er niemand,", zei ze, "en ik ben er al zo vaak geweest, 't is toch veel prettiger om er met iemand heen te gaan die er al eerder geweest is?". S. 154: "Soll ich mitgehen ins Frauenhaus?" Ich schwieg, und sie begriff, dass ich lieber alleine ging. "Du kennst da niemanden", sagte sie, "und ich bin schon oft dort gewesen. Ist es nicht einfacher, mit einer hinzugehen, die schon mal dort war?" Es geht um den letzten Satz, wo im Deutschen eine rhetorische Frage benutzt wurde ('Ist es nicht einfacher ...'), die auch sehr geeignet ist, um auf Vorangehendes zurückzugreifen.

Beim Imperativ ist der pragmatische Effekt 'Ungeduld'. Oft wurde die direktive Handlung schon vorher einmal ohne Einwilligung ausgeführt, was zu der Wiederholung führt. Ein Beispiel, S. 121: "Laat het toch verder rusten," zei ze, "laat het aan die eeuwig lange kerel over." S. 177: "Lass die Finger davon", sagte sie, "überlass das doch diesem langen Kerl". Während im Original doch im ersten der beiden Imperative steht, wurde es in der Übersetzung in den zweiten versetzt. Wenn toch im Imperativ nicht mit doch übersetzt wird, dann wird es einfach weggelassen (vier Fälle).

Die Einteilung in W-Fragen und Exklamativsätze ist problematisch, weil auch alle W-Fragen durch toch einen exklamativen Charakter bekommen. Der Hörer indiziert: Ich habe mich das schon vorher gefragt, konnte aber keine Antwort finden, und komme darum auf die Ausgangsfrage zurück. Ein Beispiel, S. 49: "Wie heeft toch je pad gekruist toen ik bij m'n moeder was? Een meisje?" S. 72: "Wer ist dir nur über den Weg gelaufen, als ich bei meiner Mutter war? Ein Mädchen?" In diesem Beispiel wurde toch mit nur übersetzt, und es fällt auf, dass in keinem der $11 \mathrm{~W}$-Fragen doch als Übersetzung vorkommt: 2-mal nur, 2mal denn, 2-mal bloß, 2-mal eigentlich, und 3-mal 'Null'. Es sieht so aus, dass wir es hier mit einem Kontext zu tun haben, der sich ohne weiteres semantisch mit toch/doch verträgt, aber in dem im Deutschen das Wort fehlt. Der Befund gibt dazu Anlass, diesen Kontext in einer kontrastiven Beschreibung hervorzuheben. Ob das auch Grund genug ist, in einer monolingualen Beschreibung eine eigene Gebrauchsweise anzunehmen, ist die Frage. Das hängt wiederum davon 
ab, was als ausreichendes Kriterium für die Unterscheidung einer Gebrauchsweise betrachtet wird. Wenn 'Satztyp' ausreicht, dann können wir mehrere Gebrauchsweisen der Modalpartikel unterscheiden. Wenn es um die Bedeutung geht, reicht die Annahme einer Gebrauchsweise, denn die spezifischen Effekte, die in den verschiedenen Satztypen auftreten (fragender Effekt im Assertivsatz, Ungeduld im Imperativsatz, exklamativer Effekt in der W-Frage) können auf der pragmatischen Ebene angesiedelt werden.

Die neun Exklamativsätze sind unterschiedlicher Form. Ein paar Beispiele, S.134: "O, mevrouw, wat kent u de misdaad toch slecht, heus, wat er allemaal niet gebeuren kan, het is maar goed dat $\mathrm{u}$ er geen weet van heeft. Maar wat jammer toch dat uw man niet praat." S. 194: "Oh, Mevrouw, wie schlecht kennen Sie sich doch im Verbrechen aus, wirklich, was nicht alles passieren kann, es ist nur gut, dass Sie keine Ahnung davon haben. Aber wie schade, dass Ihr Mann nicht redet." Der Sprecher reiht hier Ausruf an Ausruf. Im ersten und im letzten steht ein toch im Original, in der Übersetzung findet sich das erste Vorkommen, das zweite wird weggelassen. S. 145: "Ach, als het zo toch eens gegaan kon zijn!", S. 209: "Ach, wenn es sich doch so abgespielt hätte!". In zwei Fällen ist die Übersetzung 'Null', einmal wird nun gewählt, S. 180: "Jezus, dat jij toch ook aan me getwijfeld hebt". S. 261: "Jesus, dass du nun auch an mir gezweifelt hast".

Beim Ausruf kann man zwei implizite Schritte zu Grunde legen. Die Äußerung selber vertritt den positiven Schritt der Feststellung, dass es so ist. Der Sprecher indiziert: Ich habe dieses erstmals festgestellt, konnte es dann nicht glauben, aber muss es jetzt doch wohl glauben. Die Schritte sind in dieser Gebrauchsweise nicht im expliziten Dialog konstituiert, sie spielen sich innerhalb des Sprechers ab.

\section{Schlussbetrachtung}

Die Besprechung der verschiedenen Gebrauchsweisen hat gezeigt, dass alle Fälle sich gut mit der Dreischrittanalyse vertragen. Auf der Ebene der übergreifenden Bedeutung gibt es keinen Anlass, toch und doch zu unterscheiden.

Auf der Ebene der Gebrauchsweisen haben wir gesehen, dass die Unterschiede hier größer sind. Diese Unterschiede bewegen sich aber hauptsächlich auf der Ebene der Gebrauchskontexte: Im Niederländischen wird toch in W-Fragen benutzt, wo Deutsch doch diesen Kontext meidet usw. Nur in zwei Fällen gab der Gebrauchsunterschied Anlass, eine Bedeutungsspezifizierung vorzunehmen, nämlich beim Gebrauch von N. toch in der Bedeutung sowieso und bei der konjunktionalen Gebrauchsweise von doch im Deutschen (und toch sofern es noch im Niederländischen als Konjunktion verwendet wird). Im ersten Fall wird verstärkt auf Vorhergehendes zurückgegriffen, die Proposition gilt sowieso, unab- 
hängig von dem jetzigen Anlass, während im zweiten Fall gerade nicht auf Vorhergehendes zurückgegriffen wird. Bei der Konjunktion wird das Dreischrittschema zwar noch beibehalten, aber es wird für einen rein adversativen Zweck benutzt. Die von Sekiguchi postulierte, Rückkehr zur Bejahung' im 3. Schritt, ist in dieser Gebrauchsweise nicht gegeben. Wenn wir trotzdem diesen Bedeutungsaspekt in der übergreifenden Bedeutung beibehalten wollen, müssten wir zulassen, dass eine Gebrauchsweise nicht notwendigerweise alle übergreifenden Bedeutungsaspekte beinhaltet.

Was die Differenzierungen bei der Modalpartikelgebrauchsweisen betrifft (fragend im Assertivsatz, Ungeduld im Imperativ und Staunen bei der W-Frage) nehme ich an, dass diese Effekte der pragmatischen, nicht konventionalisierten Ebene zugeschrieben werden können. Die übergreifende Bedeutung wird bei der Modalpartikel doch voll realisiert.

Der Gebrauch eines Übersetzungskorpus hat sicher geholfen, die Frage der zu unterscheidenden Gebrauchsweisen empirisch zu gestalten. Vor allem wenn toch in bestimmten Fällen systematisch oder typischerweise nicht mit doch übersetzt wird, oder wenn umgekehrt doch in bestimmten Kontexten systematisch nicht auf toch zurückgeht, gab das Anlass dazu ernsthaft zu überlegen, eine eigene Gebrauchsweise anzunehmen, und sich zu fragen ob diese Gebrauchsweise eine spezifische Bedeutung aufweist. In weiterer Forschung würde ich also sicher wieder einen Korpus benutzen wollen, aber dann einen elektronisch verfügbaren Parallelkorpus; der Korpusvergleich mit der Hand, wie ich ihn jetzt vorgenommen habe, ist doch recht mühsam.

Man sollte sich der Tatsache bewusst sein, dass ein Korpus immer empirischen Beschränkungen unterliegt. Obwohl Maarten 't Hart 'natürlich' schreibt, kommen bestimmte Formen der Umgangssprache in einem solchen Korpus nicht vor. So gibt es in der niederländischen Umgangssprache noch einige Gebrauchsweisen von doch, die nicht im Korpus vertreten sind. Zwei sind zu nennen: einmal die 'tag'-Gebrauchsweise, im Sinne von 'nicht wahr?', z. B.: Dat was leuk, toch? 'Das war lustig, nicht wahr?' Dieses nachgestellte doch macht aus einer Assertion eine Vergewisserungsfrage, genau wie eine Modalpartikel im Mittelfeld. Eine zweite Gebrauchsweise kommt vor in Kombination mit einer nominalen Gruppe: "Jan toch!", direkt zu Jan gesagt, oder "die Jan toch!", 'dieser Jan doch!', wenn man über ihn spricht. Kategorial könnte man hier eine Interjektion annehmen, die Bedeutung lässt sich wie in Exklamativsätzen analysieren: die zwei impliziten Schritte sind 'sprecherintern': ich nehme etwas Erstaunliches wahr (1. Schritt), kann es nicht glauben (2. Schritt), muss es aber wohl glauben (3. Schritt).

Wenzel (2002: 295) stellte am Ende ihrer Untersuchung fest "dass die niederländischen Partikeln noch völlig unzureichend erforscht sind. Es wundert daher 
nicht, dass sie in Fremdsprachenunterricht und Lehrwerken zu kurz kommen. (...) Dabei erscheint der Vergleich mit der Forschungslage deutscher Partikeln sinnvoll, auch wenn es nicht um kontrastive Beschreibungen geht. Es ist anzunehmen, dass gerade die Ähnlichkeit die spezifischen Eigenschaften niederländischer Partikeln herausstellen kann".

Mit der hier vorgelegten kleinen vergleichenden Studie zu toch und doch wollte ich diesem Aufruf Folge leisten.

\section{Literaturangaben}

Abraham, Werner (1981): "Partikeln und Konjunktionen - Versuch einer kontrastiven Typologie Deutsch-Niederländisch". In: Weydt, Harald (ed.): Partikeln und Deutschunterricht. Heidelberg: 168-188.

Aijmer, Karin (2002): English discourse particles. Evidence from a corpus. Amsterdam.

Aijmer, Karin/Simon-Vandenbergen, Anne-Marie (to appear, 2003): "The discourse particle well and its equivalents in Swedish and Dutch". Linguistics.

Bastert, Ulrike (1985): Modalpartikel und Lexikographie. Eine exemplarische Studie zur Darstellbarkeit von DOCH im einsprachigen Wörterbuch. Tübingen.

Borst, Dieter (1985): Die affirmativen Modalpartikeln doch, ja und schon. Ihre Bedeutung, Funktion, Stellung und ihr Vorkommen. Tübingen.

Elffers, Els (1992): "Wat betekent toch toch?". In: Schermer-Vermeer, E.C. et al. (eds.): De kunst van de grammatica. Amsterdam: 63-80.

Eijirô, Iwasaki (2002): "Studien zur deutschen Sprache in Japan. Eine skizzenhafte Beschreibung ihrer Geschichte". Zeitschrift für Literaturwissenschaft und Linguistik 32 (Nr. 127): 7-18.

Ezawa, Kennosuke (1998): "Sekiguchis Sprachstudium und Grundkonzept der Grammatik". In: Harden, Theo/Hentschel, Elke (eds.): Particulae particularum. Festschrift zum 60. Geburtstag von Harald Weydt. Tübingen: 75-83.

Fischer, Kerstin/Drescher, Martina (1996): "Methods for the description of discourse particles: Contrastive analysis". Language Sciences 18: 853-861.

Foolen, Ad (1989): "Beschreibungsebenen für Partikelbedeutungen". In: Weydt, Harald (ed.): Sprechen mit Partikeln. Berlin: 305-317.

Foolen, Ad (1994): "Toch wel." In: Boogaart, Ronny/Noordegraaf, Jan (eds.): Nauwe betrekkingen. Voor Theo Janssen bij zijn vijftigste verjaardag. Amsterdam: 81-88.

Franck, Dorothea (1980): Grammatik und Konversation. Königstein/Ts.

Graefen, Gabriele (2000): "Ein Beitrag zur Partikelanalyse - Beispiel: doch." Linguistik online 6, 2. (13 Seiten)

't Hart, Maarten (1983/2001): De kroongetuige. Amsterdam. Deutsche Übersetzung von Marianne Holberg: Die schwarzen Vögel. München.

Hentschel, Elke (1986): Funktion und Geschichte deutscher Partikeln. Ja, doch, halt und eben. Tübingen.

Janssen, Theo (1995): "Deixis from a cognitive point of view". In: Contini-Morava, Ellen/Sussman Goldberg, Barbara (eds.) Meaning as explanation: Advances in linguistic sign theory. Berlin: 245-270. 
König, Ekkehard/Stark, Detlef/Requardt, Susanne (1990): Adverbien und Partikeln. Ein deutsch-englisches Wörterbuch. Heidelberg.

Kerbrat-Orecchioni, Catherine (2001): "Oui, Non, Si : un trio célèbre et méconnu". Marges Linguistique 2: 95-119. <http://www.marges-linguistiques.com>

Langacker, Ronald (to appear): "Form, meaning and behavior: The Cognitive Grammar analysis of double subject constructions". In: Kirsner, Robert S. et al. (eds.): Cognitive and communicative approaches to linguistic analysis. Amsterdam.

Meibauer, Jörg (1994): Modaler Kontrast und konzeptuelle Verschiebung. Studien zur Syntax und Semantik deutscher Modalpartikeln. Tübingen.

Métrich, R./Faucher, E./Courdier, G. (1996): Les invariables difficiles. Band 2 : baldgeradezu. 3. durchgesehene Auflage. Nancy.

Mitchell-Schuitevoerder, Rosemary (2001): When is a translation not a translation? A study of Dutch modal particles in translation and an experimental case-study. MA Thesis, University of Durham.

Ormelius-Sandblom, Elisabet (1997): Die Modalpartikeln ja, doch und schon. Zu ihrer Syntax, Semantik und Pragmatik. Stockholm.

Önnerfors, Olaf (1997): Verb-erst-Deklarativsätze. Grammatik und Pragmatik. Stockholm.

Sekiguchi, T. (1939/1977): "Was heißt doch?" In: Weydt, Harald (ed.): Aspekte der Modalpartikeln. Tübingen: 3-9.

Van Valin, Robert D. Jr. (1975): A pragmatic analysis of German doch. M.A. Thesis, University of California, Berkeley.

Wenzel, Veronika (2002): Relationelle Strategien in der Fremdsprache. Pragmatische und interkulturelle Aspekte der niederländischen Lernersprache von Deutschen. Münster.

Westheide, Henning (1989): "Zur ethnographischen Beschreibung von Partikelfunktionen am Beispiel Niederländisch-Deutsch". In: Weydt, Harald (ed.): Sprechen mit Partikeln. Berlin: 293-302.

Weydt, Harald (1969): Abtönungspartikel. Bad Homburg.

Weydt, Harald (2001): "Partikelforschung." In: Holtus, Günter et al. (Hrsg.): Lexikon der Romanistischen Linguistik. Vol. I, 1. Tübingen: 782-801.

Weydt, Harald, et al. (1983): Kleine deutsche Partikellehre. Stuttgart. 
particulae collectae 